Annuaire suisse de politique de développement

27-2 | 2008

Migration et développement: un mariage arrangé

\title{
Migration et développement : les hésitations de la politique suisse
}

Gérard Perroulaz

\section{(2) OpenEdition}

1 Journals

Édition électronique

URL : http://journals.openedition.org/aspd/521

DOI : 10.4000/aspd.521

ISSN : 1663-9669

Éditeur

Institut de hautes études internationales et du développement

Édition imprimée

Date de publication : 1 décembre 2008

Pagination : $245-260$

ISBN : 978-2-940415-07-6

ISSN : 1660-5934

\section{Référence électronique}

Gérard Perroulaz, " Migration et développement : les hésitations de la politique suisse », Annuaire suisse de politique de développement [En ligne], 27-2 | 2008, mis en ligne le 22 mars 2010, consulté le 08 septembre 2020. URL : http://journals.openedition.org/aspd/521 ; DOI : https://doi.org/10.4000/ aspd.521 


\title{
Migration et développement: les hésitations de la politique suisse
}

\author{
Gérard Perroulaz*
}

\section{Introduction}

Dans le cadre des débats actuels sur les liens entre migration et développement, plusieurs études récentes montrent que les politiques migratoires des pays du Nord ont des conséquences sur les pays en développement ${ }^{1}$. Elles soulignent les apports positifs des migrations pour ces pays dont sont originaires de nombreux migrants, et pas uniquement les aspects négatifs (fuite des cerveaux, filières criminelles pour la migration clandestine, traite des êtres humains). Par contre, le débat politique sur les migrations s'est focalisé pendant longtemps sur les questions de l'asile et de l'intégration dans les pays du Nord.

Plusieurs travaux récents, notamment ceux du Centre de développement de l'Organisation de coopération et de développement économiques (OCDE) ${ }^{2}$, relèvent l'importance d'une plus grande cohérence en faveur du développement. Il ne s'agit pas seulement d'éviter qu'une politique dans un domaine d'intervention nuise au développement, mais de parvenir à de meilleures synergies entre les diverses politiques pour en renforcer les effets positifs sur les pays d'origine des migrants. Or, deux raisons au moins empêchent la réalisation d'une cohérence parfaite entre les politiques de coopération au développement, les politiques économiques (emplois, commerce et investissements), les politiques migratoires et les politiques nationales d'intégration des étrangers. D’une part, les objectifs des différentes politiques varient. Les politiques migratoires recherchent le contrôle des flux migratoires, l'intégration des résidents étrangers, la politique d'asile et le renvoi des requérants d'asile déboutés; elles n'ont pas pour objectif prioritaire la lutte contre la pauvreté, objectif déclaré des agences d'aide au développement. D'autre part, les flux d'aide ne concernent pas les mêmes pays que les flux migratoires ${ }^{3}$ : l'aide va plutôt aux pays les plus pauvres, alors que les investissements directs à l'étranger et les flux commerciaux tendent à se concentrer dans une minorité de pays à revenu intermédiaire. La plupart des migrants qui arrivent dans les pays de l'OCDE ne proviennent pas des pays les plus pauvres; on peut en déduire que ces derniers, les plus

* Chargé de recherche à l'Institut de hautes études internationales et du développement, Genève.

1 Par exemple les recherches en cours au Centre de développement de l'OCDE. Voir aussi les travaux du Forum mondial sur la migration et le développement (FMMD).

2 Jeff Dayton-Johnson et Louka T. Katseli, Migration, aide et commerce: plus de cohérence en faveur du développement, Cahier de politique économique, $\mathrm{n}^{\circ}$ 28, Paris, OCDE, 2006.

3 Voir Denis Cogneau et Sylvie Lambert, L'aide au développement et les autres flux Nord-Sud: complémentarité ou substitution?, Document de travail, $\mathrm{n}^{\circ}$ 251, Paris, Centre de développement de l'OCDE, 2006. Voir aussi le chapitre 9 de l'ouvrage de l'OCDE La cohérence des politiques au service du développement: migrations et pays en développement (Paris, OCDE, 2007, 164 p). 
dépendants de l'aide publique au développement (APD), bénéficient relativement peu des effets positifs de la migration, tels que les envois de fonds et l'acquisition de compétences lors du retour des migrants.

Dans cet article, après une brève présentation des acteurs concernés par les migrations en Suisse ${ }^{4}$, nous évoquerons plus longuement le rôle de la Direction du développement et de la coopération (DDC). La deuxième partie de cet article se concentre sur l'analyse du discours dans le domaine du lien entre la migration et la coopération: elle relève les initiatives sur le plan international qui ont été encouragées par la Suisse et examine les textes de stratégie de la coopération. Dans la partie suivante sont exposées les difficultés pour la coopération suisse de passer des textes d'intention aux projets opérationnels en matière de migration. Nous analyserons ensuite les champs de tension entre la politique migratoire et les autres politiques de la Suisse qui ont une incidence sur le développement. Enfin, à la lumière d'études publiées au niveau international, nous examinerons le rôle plus actif que pourrait jouer la coopération, que ce soit pour réduire les effets négatifs des migrations ou pour accroître leurs effets positifs sur le développement des pays d'origine.

\section{Vue d'ensemble des acteurs concernés en Suisse}

Parmi les multiples acteurs concernés par la migration en Suisse, citons tout d'abord, au niveau de la Confédération, l'Office fédéral des migrations (ODM), qui est chargé de la politique d'asile et qui est compétent pour les questions de migration. En lien avec les instances cantonales, il a une large palette d'activités dans ce domaine, relatives aux autorisations de séjour et de travail, à la politique d'intégration, à la naturalisation, à l'asile et à l'aide au retour. Dans le domaine des affaires internationales, l'ODM a joué un rôle dans l'Initiative de Berne et dans la conclusion d'accords de réadmission avec les pays d'origine des migrants.

Outre l'ODM, le Secrétariat d'Etat à l'économie (SECO) s'est intéressé ces dernières années aux questions des transferts de fonds des migrants. La Division politique IV du Département fédéral des affaires extérieures (DFAE) s'occupe quant à elle du suivi du dialogue international dans ce domaine et de la lutte contre la traite des êtres humains. La Direction du développement et de la coopération (DDC) incluait aussi jusqu'en 2008 le thème des migrations parmi les dix thèmes prioritaires de la coopération suisse. Nous verrons que les projets de la DDC concernent en fait surtout l'aide humanitaire.

Il faut finalement relever le rôle de la Commission fédérale contre le racisme ${ }^{5}$ et de la Commission fédérale pour les questions de migration ${ }^{6}$. Cette dernière commission extraparlementaire, née de la fusion de l'ancienne Commission fédérale des étrangers et de celle des réfugiés, a été instituée par le Conseil fédéral le $1^{\text {er }}$ janvier 2008.

4 D'autres contributions de ce dossier décrivent l'action de certains organes gouvernementaux. Voir notamment le texte de Pietro Mona (Division politique IV Sécurité humaine, Département fédéral des affaires étrangères) et celui d'Eric Kaser et Saskia Schenker (Office fédéral des migrations, Département fédéral de justice et police).

$5<$ http://www.ekr-cfr.ch>.

$6<$ http://www.ekm.admin.ch>. 
La Suisse a participé au dialogue international sur les migrations et soutient le travail de plusieurs organisations internationales actives dans ce domaine: le Haut-Commissariat pour les réfugiés (HCR), le Haut-Commissariat aux droits de l'homme (HCDH), l'Organisation internationale du travail (OIT) pour ce qui a trait aux droits et conditions de travail des migrants, et l'Office de secours et de travaux pour les réfugiés de Palestine (UNRWA). La Confédération a des liens particulièrement étroits avec l'Organisation internationale pour les migrations (OIM), basée à Genève, dont elle finance le bureau à Berne et avec laquelle elle collabore activement. Cette entité est notamment mandatée pour mettre en œuvre les programmes d'aide au retour et établir des partenariats migratoires.

Parmi les autres acteurs, les cantons jouent un rôle très important, notamment pour l'octroi des permis de travail et des régularisations de sans-papiers. Les migrants - les premiers concernés - sont aussi des interlocuteurs par l'intermédiaire de leurs associations. Quant aux organisations non gouvernementales (ONG), elles mènent des actions humanitaire lors de catastrophes et de conflits, mais promeuvent également les droits de l'homme et le droit d'asile en luttant contre le durcissement des procédures d'accueil des requérants d'asile en Suisse et en apportant à ces dernier un appui concret et un conseil individuel pour l'aide au retour. La Croix-Rouge suisse, Caritas, l'Entraide protestante (EPER), la Fondation Terre des hommes, le Centres social protestant figurent parmi les principales ONG actives dans le domaine des migrations. Certaines ONG, telles l'organisation faîtière OSAR, Solidarités sans frontières ou la plate-forme sur Internet $<$ http://www.humanrights.ch $>$, sont spécialisées dans le domaine du plaidoyer en faveur du droit d'asile ou des étrangers; d'autres, nombreuses, s'attachent à l'intégration, comme par exemple le Centre de contact Suisses-Immigrés.

\section{Le rapprochement entre migration et coopération et la participation suisse au dialogue international}

Dès la fin des années 1980, quelques initiatives ont timidement commencé à associer coopération internationale et migration, alors qu'auparavant l'immigration des travailleurs étrangers en Suisse, les procédures d'asile et l'aide humanitaire aux réfugiés dans les régions en crise avaient été gérées séparément. On peut dater précisément la première apparition en Suisse du lien entre coopération et migration, dans un postulat du conseiller national Ernst Mühlemann ${ }^{7} \mathrm{du}$ 6 décembre 1988 qui demandait au Conseil fédéral d'accorder une priorité accrue à la coopération au développement avec la Turquie. En effet, à la suite d'un brusque afflux de requérants d'asile d'origine turque, il fallait trouver les moyens d'en rapatrier le plus grand nombre ${ }^{8}$. La guerre dans les Etats de l'ancienne Yougoslavie permit ensuite de renforcer la relation entre politique d'asile et aide humanitaire: celle-ci devait contribuer toujours davantage à maintenir les populations dans leur pays (aide aux déplacés internes) ou dans les pays environnants, afin de freiner l'arrivée de réfugiés en Europe occidentale. L'argument

7 Conseiller national radical de 1983 à 1999 (Thurgovie) et membre de la Commission de politique extérieure de 1995 à 1999.

8 DDC, Coopération internationale et migrations, Ecrits sur le développement, $\mathrm{n}^{\circ} 4$, Berne, DDC, avril 2001. 
économique est aussi avancé: le coût annuel lié à la présence de requérants d'asile en Suisse est bien plus élevé que les dépenses pour l'accueil de réfugiés dans des camps des régions ou pays limitrophes des zones en crise.

Dans les années 1990, le besoin d'une meilleure coordination entre les offices chargés des questions migratoires se fit sentir. Le Conseil fédéral créa en 1992 le Groupe de travail interdépartemental pour les problèmes de migration, devenu Groupe de travail interdépartemental pour les questions de migration en 1998 (Groupe IAM), dans lequel sont représentés tous les départements fédéraux. En 1995 fut créé le Groupe directeur interdépartemental d'aide au retour, sous la direction conjointe de l'ODM et de la DDC et avec des représentants de la DP IV et de l'OIM.

\section{Une coopération internationale indispensable}

Si les politiques migratoires sont un élément très important de la souveraineté nationale de chaque Etat, une meilleure concertation internationale s'avère cependant indispensable pour encadrer et gérer les flux migratoires ${ }^{9}$. La Suisse a largement encouragé cette concertation internationale, avec le lancement en 2001 de l'Initiative de Berne par l'Office fédéral des réfugiés (aujourd'hui ODM). Dans ce cadre, deux conférences internationales organisées à Berne en 2003 et 2004 permirent d'entamer un dialogue entre les pays d'origine, de transit et de destination des migrants ${ }^{10}$ et contribuèrent à la finalisation de l'Agenda international pour la gestion des migrations, qui pose les grandes lignes de la coopération internationale dans ce domaine et reconnaît l'importance de la renforcer. Sans force contraignante, cet agenda international est le résultat de consultations, et non de négociations entre Etats.

Parallèlement à ce processus entamé par la Suisse, Kofi Annan, ancien secrétaire général des Nations unies, proposa en 2002 déjà que soit menée au niveau des Nations unies une réflexion sur les migrations. Un groupe de travail de l'ONU releva dans un rapport publié en 2003 les lacunes institutionnelles et l'insuffisance des normes internationales dans ce domaine (rapport Doyle) ${ }^{11}$. Il examinait notamment le rôle que pourraient jouer les organisations internationales et proposait quelques variantes pour l'avenir, dont la possibilité de créer une agence internationale qui coordonnerait les activités des Nations unies dans ce domaine, les organisations existantes ne s'occupant que partiellement de cet aspect (HCR, OIT et OIM, celle-ci avec un statut un peu particulier car elle ne fait pas partie du système des Nations unies) ${ }^{12}$.

A la suite du rapport Doyle, la Commission mondiale sur les migrations internationales (CMMI) fut créée en décembre 2003, en présence de la conseillère

9 Voir notamment DDC, op. cit., ou la revue Un seul monde de la DDC, nº 2, 1999.

10 Sur l'Initiative de Berne, voir, sur le site de l'ODM, <http://www.bfm.admin.ch/bfm/fr/home/themen/ internationales.html > >affaires multilatérales.

11 Michael Doyle, Report to the Secretary General on Migration, New York, 2003, 41 p.

12 Le rapport Doyle mentionnait d'autres options possibles, comme la désignation d'une agence existante pour coordonner les actions dans le domaine des migrations, l'intégration de l'OIM dans le système des Nations unies comme agence spécialisée, l'adoption d'accords internationaux de régulation des migrations (à l'instar des accords commerciaux) ou l'instauration d'une commission internationale. 
fédérale Micheline Calmy-Rey. Cette commission d'experts, présidée par le Suisse Rolf K. Jenny, publia le 5 octobre 2005 son rapport final ${ }^{13}$, dans lequel elle relevait que pour accroître au maximum les bénéfices des migrations internationales et réduire au minimum leurs effets négatifs, les politiques migratoires devaient être fondées sur des objectifs partagés et une vision commune. En outre, la commission proposait six principes d'action: migrer par choix, renforcer l'impact sur l'économie et le développement, aborder la question de la migration irrégulière, renforcer la cohésion sociale par l'intégration, protéger les droits des migrants et, enfin, renforcer la gouvernance; ces principes étaient assortis de recommandations qui restent pleinement d'actualité.

La réflexion se poursuivit sur le plan international en septembre 2006 lors du Dialogue de haut niveau des Nations unies sur les migrations internationales et le développement. Cette conférence confirma la nécessité de poursuivre le dialogue entre les Etats; dans ce dessein, le secrétaire général de l'ONU proposa la création du Forum mondial sur la migration et le développement (FMMD), plutôt que d'instaurer une nouvelle institution internationale ou de désigner l'une des organisations existante comme coordinatrice des activités dans ce domaine. Ce forum, plus informel, est basé sur la participation volontaire des Etats et l'implication de la société civile. Il n'a pas pour objectif de produire des résultats négociés ou des décisions contraignantes, mais ouvre un espace de dialogue et d'échange d'expériences et de bonnes pratiques. Les représentants de 150 Etats participèrent à sa première réunion, tenue à Bruxelles en juillet 2007, et une seconde réunion se déroule à Manille en octobre 2008 ${ }^{14}$. La Suisse contribue activement aux travaux du FMMD par l'entremise de la DP IV du DFAE. Des points focaux nationaux devraient permettre d'adopter une approche plus complète et plus cohérente de la migration et du développement.

\section{L'aide, un moyen de freiner les migrations?}

Autant la Suisse a été à l'origine d'initiatives intéressantes pour stimuler le dialogue sur le plan international, autant elle a de la peine à traduire certaines propositions dans des actions de coopération bilatérale. La DDC s'est montrée relativement défensive dans ce domaine, arguant que l'APD ne saurait empêcher les flux migratoires et s'opposant à la conditionnalité de l'aide (par exemple la suppression de l'aide aux pays non coopérants lors du renvoi de leurs ressortissants) et à son instrumentalisation à des fins telles que le renvoi massif d'étrangers. Cantonnée dans cette attitude, la DDC n'a pas été capable de promouvoir suffisamment de projets qui viseraient à maximaliser certains aspects positifs des migrations alors que des pistes intéressantes sont évoquées dans de nombreuses recherches et études. Ainsi, tout en prônant, dans plusieurs documents publiés autour de l'an 2000, la coopération au développement comme un outil de lutte efficace contre certaines causes de migration ${ }^{15}$, la DDC n'a cessé de

13 Commission mondiale sur les migrations internationales (CMMI), Les migrations dans un monde interconnecté: nouvelles perspectives d'action, 2005, <http://www.gcim.org>.

14 Rapport de la $1^{\text {re }}$ session du Forum sur la migration et le développement, Bruxelles, 9-11 juillet 2007, $30 \mathrm{p}$.

15 Le document le plus complet de la DDC sur les questions migratoires est Coopération internationale et migrations, op. cit. Voir aussi le dossier sur les migrations de sa revue Un seul monde, $\mathrm{n}^{\circ} 2,1999$. 
préciser en parallèle que la coopération au développement n'était pas en mesure de freiner les flux migratoires, quand bien même elle concentrerait toute son aide sur les pays d'origine des migrants en Suisse ${ }^{16}$.

En 2003, dans son message sur la continuation de la coopération technique et de l'aide financière en faveur des pays en développement, le Conseil fédéral relevait le point suivant: «La principale contribution de la coopération au développement à la résolution du problème de la migration est de s'attaquer à long terme aux causes de la migration forcée et par nécessité, et ce principalement en aidant à réduire la pauvreté et, partant, à exercer un effet préventif général sur la migration. L'amélioration de l'accès à l'emploi, à la formation et à la santé, à l'eau potable, ainsi que la possibilité de participer sans entraves à l'activité politique, contribuent, au même titre qu'une gestion durable des ressources existantes, à faire en sorte qu'aux yeux d'un grand nombre de personnes l'émigration n'est plus une solution qui se justifie. ${ }^{17}$ Les migrations sont ainsi considérées comme étant fondamentalement un «problème» alors qu'il est généralement admis qu'elles sont une réalité incontournable et qu'elles se poursuivront ces prochaines décennies.

Dans le même message, le Conseil fédéral reconnaît cependant que les montants modestes fournis par la Suisse au titre de la coopération n'arrêteront pas les flux migratoires, que les mesures isolées prises par les pays du Nord ne peuvent résoudre le problème des migrations incontrôlées et qu'une action concertée sur le plan international est nécessaire. Concernant la réadmission des ressortissants par les Etats, le message relève: «Vouloir lier la coopération au développement avec les problèmes qui se posent dans ce domaine et la suspendre ou y mettre un terme dans les Etats qui ne coopèrent pas de manière suffisante ou refusent de coopérer, n'est ni réaliste ni constructif. ${ }^{18}$ Le Conseil fédéral s'oppose ainsi à la conditionnalité de l'aide. Pour le Conseil fédéral, il n'est pas judicieux de recommander la concentration de l'aide dans les principaux pays de provenance des migrants - d'autant plus que ces pays sont dans l'ensemble des pays à revenu intermédiaire alors que l'aide suisse est destinée prioritairement aux pays les plus pauvres.

\section{Message sur la coopération suisse 2008-2012: les migrations reléguées à l'arrière-plan}

Malgré le foisonnement d'études sur le plan international qui soulignent les liens entre migration et développement, le nouveau message du Conseil fédéral sur la continuation de la coopération technique et de l'aide financière en faveur des pays en développement fait une description plutôt sommaire du phénomène de la migration: "Quelque 200 millions de personnes, soit près de 3\% de la population mondiale, vivent loin de chez elles, et leur nombre tend à s'accroître. Des raisons d'ordre économique, mais aussi les conflits politiques et sociaux, poussent

16 Les études de l'OCDE montrent que la corrélation entre l'aide et la croissance économique est faible et que la croissance économique au Sud n'entraîne pas (sauf à très long terme) une diminution des flux migratoires.

17 Message concernant la continuation de la coopération technique et de l'aide financière en faveur des pays en développement du 28 mai 2003 (Feuille fédérale [FF] 2003: 4155), p. 4189

18 Ibid., p. 4190. 
nombre de personnes à émigrer vers les pays industrialisés ou émergents. Les flux migratoires Sud-Sud demeurent cependant les plus denses. Les migrations ont donné naissance à de grandes communautés étrangères dans les pays d'immigration, un phénomène qui recèle aussi bien des risques (problèmes d'intégration et exode des cerveaux, par exemple) que des chances (transfert de savoir-faire et envoi d'argent des émigrés vers leur pays d'origine). Le volume des transferts financiers que ces diasporas opèrent vers leurs pays d'origine équivaut à plusieurs fois le budget total de la coopération au développement. L'un des défis pour la coopération consiste dès lors à créer des incitations pour exploiter ce potentiel. ${ }^{19}$

La migration, qui faisait partie des dix thèmes prioritaires de la DDC dans le crédit-cadre de 2003, ne figure plus parmi les sept thèmes prioritaires de la DDC dans le nouveau message. Celui-ci relève toutefois que la DDC peut s'occuper également de coopération régionale, de prévention des conflits et de migration dans les 12 pays de concentration de l'aide suisse. En juin 2008, la conseillère fédérale Micheline Calmy-Rey a estimé que des évolutions globales telles que la crise alimentaire, le manque d'eau, les migrations et les Etats en échec constituaient des facteurs déclencheurs pour la grande réorganisation de la DDC entamée ce même été ${ }^{20}$, et un processus a été lancé au sein du DFAE pour préparer trois propositions de stratégie dans trois domaines jugés prioritaires: le changement climatique, l'alimentation et les migrations. Or, lors de la Conférence annuelle de la DDC et du SECO en août, la cheffe du DFAE n'a finalement présenté les actions que dans deux domaines: la sécurité alimentaire et le changement climatique ${ }^{21}$. En fait, comme nous le verrons ci-dessous, les projets opérationnels dans le domaine des migrations ont de tout temps été d'une portée assez modeste par rapport à ceux d'autres thèmes prioritaires.

\section{Un passage aux actes difficile}

Même si la Suisse a participé très activement à la réflexion internationale sur les liens entre migration et développement, ce thème est difficilement descendu au niveau des sections opérationnelles. Dans le domaine des migrations, ce sont surtout les sections s'occupant de l'aide aux pays de l'Europe du Sud-Est qui ont développé des projets - souvent liés à l'aide au retour - dans les principaux champs d'action suivants :

- Programmes d'aide humanitaire à l'intention des réfugiés et des personnes déplacées de l'intérieur dans diverses régions en crise. En 2006 et 2007, les régions d'intervention de la DDC ont été essentiellement la Bosnie-et-Herzégovine, le Proche-Orient (aide aux réfugiés palestiniens), l'Arménie, la Thaïlande (aide aux réfugiés birmans), l'Afghanistan (aide au retour), l'Irak (aide aux déplacés internes et aide au retour), la Colombie (aide aux déplacés internes), l'Algérie (aide aux réfugiés sahraouis) et le Tchad (aide aux réfugiés provenant du Darfour). cière en faveur des pays en développement du 14 mars 2008 (FF 2008 2595), pp. 2602-2603.

20 DFAE, communiqué de presse, 9 juin 2008.

21 DDC, Conférence annuelle de la coopération au développement, communiqué de presse, 22 août 2008. 
$\checkmark$ Aide aux migrants dans les pays de transit. Prenons l'exemple du Maroc, qui sert de pays de transit pour un nombre croissant de migrants originaires d'Afrique subsaharienne cherchant à venir en Europe. Ces migrants de passage en situation irrégulière ne bénéficient pas de protection juridique et ne parviennent pas à assurer leurs besoins vitaux en nourriture et en logement. Les projets d'aide d'urgence, réalisés avec la collaboration d'ONG telles que Caritas, assurent les besoins alimentaires de base, des soins médicaux, l'hébergement et un soutien psychologique. Médecins du Monde fournit des soins médicaux et une assistance juridique et l'OIM propose une aide au retour au pays d'origine.

- Aide au retour. La DDC participe aux programmes d'aide au retour, et notamment à l'aide structurelle sur place, avec le financement et la collaboration de l'ODM.

- Programmes de prévention des migrations irrégulières. Ceux-ci comprennent en particulier des pièces de théâtre montées en Moldavie, en Biélorussie et en Ukraine, afin de sensibiliser les jeunes femmes et leurs familles aux risques liés à la migration irrégulière.

- Aide sur place aux victimes du trafic d'êtres humains. Un soutien financier est apporté aux autorités locales et à des ONG pour la réintégration de victimes de la traite dans leur pays d'origine (prise en charge médicale, appui psychologique, formation et aide au retour). Ces programmes se déroulent surtout dans les Balkans, en Russie, en Moldavie, en Ukraine et en Biélorussie.

Nous ne disposons pas de données précises sur les montants d'aide publique au développement destinés à financer des projets dans le domaine des migrations, et il est en outre difficile d'en faire une estimation tant ce domaine est peu clairement délimité. Par exemple, un programme créateur d'emplois dans une région défavorisée aura un effet indirect positif sur la migration en ce qu'il offre une perspective d'emploi à des personnes qui seraient sinon tentées de migrer. Cependant, à partir des données fragmentaires disponibles sur les projets de la DDC, nous pouvons dégager trois constats au sujet de l'impact de ces projets sur la migration.

1. Les principaux programmes d'aide dans le domaine des migrations concernent l'aide humanitaire. Celle-ci représente environ un cinquième du total de l'APD, les $80 \%$ restants étant des projets de coopération au développement à plus long terme. D'après l'information disponible, cette proportion semble s'inverser dans le domaine des migrations, où la plupart des projets de la DDC consistent dans l'aide d'urgence (aux personnes déplacées internes et réfugiés dans les pays voisins), l'aide au retour pour les requérants d'asile déboutés et la lutte contre le trafic d'êtres humains. Dans ses textes, la DDC avance souvent l'argument qu'aider les réfugiés et les personnes déplacées sur place ou dans la région d'une crise est préférable au risque de voir des flots de réfugiés arriver dans les pays du Nord. L'argument économique est aussi avancé, car il est moins cher de secourir un réfugié dans un pays voisin du conflit que de subvenir aux besoins d'un requérant d'asile en Suisse.

2. La majeure partie des dépenses intégrées dans l'APD n'a rien à faire avec une véritable aide au développement dans le domaine des migrations. En 2007, l'aide publique au développement fournie par la Suisse s'élevait à 2020 millions 
de francs, dont 9,3 millions de francs versés par l'ODM au titre de l'aide au retour et 182,9 millions de francs de dépenses en Suisse (coûts liés à la présence de requérants d'asile en Suisse lors de leur première année de séjour en Suisse) $)^{22}$. Si l'aide au retour peut être considérée comme une aide au développement dans le cas de l'aide structurelle sur place, les dépenses liées à la présence du requérant d'asile en Suisse n'ont pas d'impact sur le développement de son pays d'origine.

3. Quant aux projets développés par la DDC dans le domaine de la coopération au développement à plus long terme, ce sont le plus souvent des projets pilotes, d'un montant très modeste ${ }^{23}$.

Ainsi, l'aide au développement est encore trop souvent considérée comme un moyen de freiner les migrations et de lutter contre le trafic d'êtres humains; quant à la migration, elle est considérée avant tout comme un problème en soi, contre lequel on doit lutter. Trop peu de projets opérationnels existent pour renforcer les effets positifs des migrations et l'apport des migrants tant à leur pays d'origine qu'à leur pays d'accueil. A la fin de cet article, nous donnerons quelques pistes pour des actions qui soient plus étroitement liées à la coopération au développement à plus long terme.

\section{Tensions entre la politique migratoire et d'autres politiques suisses}

Il est inévitable que des champs de tension surgissent entre les divers départements concernés par les questions migratoires, et même à l'intérieur des différents offices. Nous relevons ci-dessous quelques-unes des contradictions entre politique d'asile, politique migratoire, politique humanitaire et politique de protection des droits de l'homme.

\section{Une politique d'admission à deux vitesses}

Depuis l'instauration de la politique des «trois cercles» en 1991 et dans toutes les adaptations ultérieures des lois concernées, la politique migratoire a privilégié les migrants qualifiés en provenance des pays européens. Depuis l'entrée en vigueur de l'Accord bilatéral avec l'Union européenne sur la libre circulation des personnes le $1^{\text {er }}$ juin 2002 sont accueillies en priorité en Suisse les personnes provenant des pays de l'Union européenne (et de l'AELE) de toutes qualifications, pour autant qu'elles trouvent un emploi en Suisse. L'accès au marché suisse du travail devient difficile pour les personnes qualifiées provenant des Etats tiers et la porte reste fermée aux personnes peu qualifiées des pays extra-

22 Chiffres de la DDC; voir <http://www.deza.admin.ch/fr/Accueil/La_DDC/Chiffres_et_faits>. Rappelons, à titre comparatif, qu'en 2005 les montants transférés dans leur pays par les travailleurs étrangers en Suisse étaient estimés à 9 milliards de dollars (y compris les transferts dans les pays voisins et ceux des frontaliers)

23 Citons l'exemple du projet Re-connect, d'un montant de 150000 francs, qui s'est déroulé en Bosnie de 2005 à 2007. Il s'adressait aux jeunes Bosniaques vivant en Bosnie-et-Herzégovine ou à l'étranger qui venaient de terminer leurs études. Les participants pouvaient effectuer des stages en Bosnie, ce qui permettait d'acquérir une certaine expérience professionnelle, de prévenir l'émigration et, pour des jeunes de la diaspora en Suisse, d'envisager un retour au pays grâce à une meilleure connaissance des conditions sur place. 
europérens. Les ressortissants de pays pauvres désireux de trouver un travail et d'appuyer leur famille dans leur pays d'origine n'ont ainsi plus d'autre choix que d'essayer de venir en Suisse par des voies illégales. Le corollaire de cette fermeture est le développement d'une économie clandestine qui, selon des estimations, occupe près de 100000 travailleurs sans statut légal ${ }^{24}$. Leur présence répond à des besoins réels de l'économie suisse en personnel peu qualifié dans les secteurs de l'économie domestique, l'hôtellerie/restauration et l'agriculture.

Cette politique d'admission ne prend pas en compte les résultats de recherches récentes, comme celles de l'OCDE, qui mettent en évidence certains effets positifs des migrations sur les pays d'origine les plus pauvres. Plusieurs études montrent en particulier que les migrants les moins qualifiés ont tendance à envoyer d'avantage de fonds dans leur pays d'origine que les émigrés hautement qualifiés ou installés depuis longtemps dans un pays du Nord ${ }^{25}$.

\section{Une politique d'asile de plus en plus restrictive}

Depuis plusieurs décennies, le contexte politique en Suisse est marqué par le durcissement progressif de la politique d'accueil des réfugiés. En effet, sous la pression des votes populaires et de l'agenda politique des partis nationalistes, la politique d'asile est devenue l'une des plus restrictives d'Europe ${ }^{26}$. Nous ne développerons pas ici cet aspect mais renvoyons le lecteur intéressé à la contribution de Gianni D’Amato dans ce volume.

\section{Un climat politique tendu}

Les ONG de défense du droit d'asile n'ont eu de cesse de dénoncer des dérives racistes et des violations des droits de l'homme en Suisse, comme des actes violents contre des étrangers ou des dérapages lors du renvoi de requérants d'asile déboutés. Certains milieux nationalistes et certains médias n'hésitent pas à pointer du doigt l'une ou l'autre communauté, attisant des tendances xénophobes et racistes. La perception vis-à-vis d'une communauté étrangère peut heureusement changer avec le temps. Les Sri Lankais, dont la présence suscitait une grande méfiance au milieu des années 1980, ont fini par être appréciés pour leur sérieux et leur ardeur au travail ${ }^{27}$. Mais le climat politique en Suisse - à l'instar des autres pays européens - n'est pas favorable à l'ouverture et le débat politique est souvent plus émotionnel que factuel.

24 Voir Claude Longchamp et al., Sans Papiers in der Schweiz: Arbeitsmarkt, nicht Asylpolitik ist entscheidend. Schlussbericht im Auftrag des Bundesamtes für Migration, Bern, GFS, Februar 2005.

25 Voir le chapitre 4 de l'ouvrage de l'OCDE La cohérence des politiques au service du développement: migrations et pays en développement, op. cit.

26 Pour suivre année après année la chronique du renforcement des lois, voir les chapitres sur la politique migratoire et les questions d'asile de l'Annuaire Suisse-Tiers Monde (années 1984-2002) puis du volume Faits et statistiques de l'Annuaire suisse de politique de développement (années 20022008).

27 Voir par exemple l'analyse de Joëlle Moret, Denise Efionayi et Fabienne Stants, Diaspora sri lankaise en Suisse, Berne, ODM, 2007, 156 p. 
Comme les autres pays européens, la Suisse a des difficultés à exécuter le renvoi des requérants d'asile déboutés. Devant la nécessité de parvenir à une politique cohérente et efficace de tous les départements et offices concernés par le domaine de la migration et du retour, le Conseil fédéral a mis sur pied en 2003 le groupe de travail interdépartemental «Politique extérieure dans le domaine de la migration et du retour» (IDAG Migration), réunissant l'ODM et la DP IV, dont les travaux ont donné lieu à un rapport publié l'année suivante ${ }^{28}$. Celui-ci confirme l'engagement de la Suisse dans le dialogue international et met en avant la nécessité de conclure des partenariats migratoires et en particulier de poursuivre les accords de réadmission avec les principaux pays d'origine des migrants. Une quarantaine d'accords bilatéraux de réadmission ont ainsi été conclus par la Suisse ${ }^{29}$. En 2003, la conseillère fédérale Ruth Metzler était allée plus loin en cherchant à négocier un accord de transit avec le Sénégal: celui-ci aurait permis que des requérants d'asile africains soient renvoyés sur son territoire en attendant de déterminer leur nationalité exacte et de pouvoir les renvoyer dans leur pays d'origine. Mais ces efforts n'aboutirent pas car, à la suite de la mobilisation de la société civile, le Parlement du Sénégal refusa de ratifier ce type d'accord.

\section{L'aide au retour vraiment volontaire?}

L'instrument de l'aide au retour volontaire est considéré par la Suisse comme exemplaire. Mais peut-on vraiment parler d'une aide au retour «volontaire»? Les personnes concernées, soit la majorité des requérants d'asile déboutés, n'ont pas de véritable choix: elles peuvent soit disparaître dans la clandestinité, soit tenter leurs maigres chances dans un autre pays européen, soit risquer un renvoi forcé dans leur pays... ou accepter une aide et retourner chez elles.

L'aide au retour s'est avérée un instrument exemplaire dans un contexte bien particulier, celui de la fin du conflit dans les Etats de l'ancienne Yougoslavie. Ainsi, sur les 65000 personnes qui ont bénéficié de l'aide au retour, 40000 provenaient du Kosovo et 10000 de Bosnie. Or, des programmes d'aide au retour existent pour une vingtaine de pays, avec dans certains cas quelques centaines de «candidats» seulement. L'instrument est-il vraiment adapté à d'autres contextes qu'une région au sortir d'une guerre? Comment en effet espérer se réinsérer durablement dans une région en proie par exemple à un taux de chômage élevé ou à des conflits interethniques latents?

Les études de l'ODM mettent en avant les synergies entre l'aide individuelle qu'il fournit et les projets d'aide structurelle sur place menés par la $\mathrm{DDC}^{30}$. Ces

28 Le rapport final est disponible en allemand: IDAG Migration, Schlussbericht der IDAG Migration, Bern, 2004. Un résumé existe en français à l'adresse suivante: <http://www.eda.admin.ch/etc/ medialib/downloads/edazen/topics/migr.Par.0003.File.tmp/Zusa_Schlussbericht_Interdept_AG_ Migration_fr.pdf $>$.

29 La liste de ces accords est disponible à la page <http://www.bfm.admin.ch/bfm/fr/home/themen/ internationales/internationale_vertraege/ref_schweizerische.html>.

30 Voir le site Internet de l'ODM, <http://www.bfm.admin.ch>, pour les rapports d'évaluation de divers programmes d'aide au retour. 
synergies entre aide au retour et coopération au développement semblent effectives dans les pays où la DDC est déjà bien présente et où elle dispose d'une palette étendue d'instruments et de contacts locaux; par contre, elles paraissent plus difficiles à établir dans les pays où la DDC a peu de programmes en cours.

\section{L'instrumentalisation de l'aide pour freiner la migration}

Le risque est grand de vouloir utiliser l'instrument de l'aide au développement pour forcer les pays partenaires à réadmettre leurs ressortissants déboutés par la Suisse. Soumettre l'APD à la condition d'une «bonne pratique de réadmission» a été dénoncé à plusieurs reprises.

A la question de savoir si ces dernières années les pressions avaient augmenté pour que l'aide soit dirigée prioritairement dans certains pays ou certains domaines répondant à des critères politiques, Walter Fust, ancien directeur de la DDC, a répondu dans une interview récente: «Indiscutablement. Cela n'est pas un problème s'il y a un débat sur la question. Mais la lutte contre la pauvreté doit demeurer la priorité. En revanche, la coopération perd très rapidement de sa crédibilité lorsque les pressions augmentent pour instrumentaliser la coopération au développement au service d'autres objectifs.» Et de préciser: «Par exemple en politique migratoire, si quelques centaines de personnes doivent être renvoyées dans un pays et que l'on doive en faire un pays prioritaire en raison de l'aide au retour, cela n'a rien à faire avec la lutte contre la pauvreté. De même si nous nous engageons dans une quelconque initiative juste pour faire plaisir à une grande puissance. Nous plaidons pour une aide qui ne varie pas au gré des occasions, mais qui soit fondée sur la qualité et la continuité. On peut recourir aux prestations de l'aide à des fins de politique extérieure ou migratoire, tant qu'on ne les détourne pas de leur objectif [de réduction de la pauvreté]. ${ }^{31}$

\section{Pistes d'action pour une coopération internationale plus active}

Le rapport de la Commission mondiale sur les migrations souligne l'importance de politiques qui permettraient de lutter contre les conséquences négatives de la migration tout en cherchant à en maximaliser les effets positifs dans les pays d'origine des migrants. Il s'agit non pas d'instrumentaliser la coopération pour freiner les migrations, mais plutôt de favoriser les migrations pour le développement.

Dans le domaine migratoire, la coopération suisse, comme celles d'autres pays de l'OCDE, développe des projets visant surtout à freiner l'arrivée des migrants (prévention des migrations, aide au retour), à lutter contre la traite des êtres humains et encore à répondre à des situations de crise par des actions humanitaires. Il n'existe à ce jour que très peu de projets basés sur une vision plus positive des migrations et sur de nouvelles formes d'appui des communautés des migrants. Voici quelques pistes qui pourraient être à l'origine de ce genre de projets.

31 «Entwicklungshilfe darf nicht instrumentalisiert werden», NZZ, 22. April 2008 (notre traduction). 


\section{Soutenir les initiatives des associations de migrants}

Un des principes fondamentaux de la coopération au développement est d'être à l'écoute des besoins de la base. Cependant, dans les programmes de la Suisse relatifs aux migrations, les initiatives ont souvent été prises «du haut vers le bas », comme par exemple les projets d'aide au retour lancés par l'ODM. Pour être attentif aux besoins de la base, il faudrait déjà mieux connaître les communautés de migrants en Suisse et identifier avec eux leurs besoins; or les associations de migrants, les ONG et les agences officielles de coopération ne se connaissent pas beaucoup. Qu'en est-il des besoins prioritaires des migrants en Suisse? Comment réduire les coûts des transferts monétaires à leurs familles? Quid de l'aide au retour? C'est seulement sur la base d'études permettant de mieux identifier les besoins réels que des projets adaptés pourront être développés. D'ailleurs, plutôt qu'une relation directe entre l'Etat et les associations de migrants, ce sont les ONG ou les fédérations cantonales de coopération qui pourraient s'avérer les mieux placés pour élaborer des projets en collaboration avec les associations de migrants - ce qui n'exclut pas des formes de soutien de la Confédération ou de cantons à des initiatives de la diaspora. En outre, lors de sa réunion à Bruxelles, le FMMD a recommandé l'établissement de partenariats tripartites entre diasporas, pays d'origine et pays d'accueil.

\section{Compenser les politiques migratoires encourageant la fuite des cerveaux}

Selon l'OCDE, certaines politiques des pays de l'OCDE ne tiennent pas compte de leurs effets sur les pays en développement. D'après des chiffres avancés par l'OIM, un médecin africain sur quatre travaille dans un pays de l'OCDE, alors que beaucoup de pays africains manquent de ressources humaines dans leurs systèmes de santé. Les pays de l'OCDE ont souvent encouragé cette fuite des cerveaux par l'octroi de permis de travail ciblant les professionnels de santé qui manquent dans les hôpitaux du Nord. S'il est difficile d'inverser la tendance et d'empêcher qu'un médecin égyptien préfère travailler dans une permanence à Genève plutôt qu'au Caire, la perte pour le pays d'origine pourrait être compensée par d'autres moyens, tels qu'une aide ciblée à son secteur de la santé et le renforcement de ses capacités de formation, ou encore la mise à disposition de compétences en permettant au médecin d'aller y travailler temporairement.

\section{Imaginer de nouvelles formes de migration temporaire et circulaire}

Il est important de pouvoir offrir des possibilités de séjour temporaire en Suisse aux travailleurs souhaitant y exercer des activités professionnelles et aux étudiants désireux d'y acquérir une expérience professionnelle avant de retourner chez eux. A Bruxelles, le FMMD a relevé que des accords conjoints entre les pays d'origine et de destination aideraient à améliorer la protection des droits des travailleurs temporaires, notamment des migrants les moins qualifiés, et à améliorer ainsi leurs contributions dans leur pays d'origine. Les pays de destination pourraient par exemple adopter des mesures plus flexibles pour le droit d'entrée et l'obtention d'un permis de travail, et en échange les pays d'origine s'engageraient à inciter les migrants à utiliser leurs compétences dans leur pays. 
Le débat sur de nouvelles formes d'accueil temporaire à imaginer risque toutefois d'être difficile à mener en Suisse compte tenu de l'ancien statut de saisonnier, très controversé.

\section{Utiliser les compétences des migrants pour des projets dans les pays d'origine}

Plusieurs programmes de coopération sont destinés à favoriser la mise à disposition temporaire de compétences de résidents étrangers dans les pays du Nord pour appuyer des projets au Sud. Un médecin pourrait ainsi enseigner et travailler temporairement dans son pays d'origine. Les projets MIDA («Migration pour le développement en Afrique») de l'OIM mobilisent par exemple la diaspora africaine en Europe au travers d'affectations temporaires dans la région des Grands Lacs ou au Ghana et au Sénégal ${ }^{32}$. De même, le programme TOKTEN ( «Transfert of Knowledge through Expatriate Nationals») du Programme des Nations unies pour le développement (PNUD) encourage le transfert des connaissances à l'aide des nationaux expatriés.

Si des évaluations doivent encore prouver que ce genre d'initiatives est à même d'avoir un impact sensible sur le développement du pays partenaire au Sud, par exemple en améliorant sa situation sanitaire, et même si ces programmes ne sauraient remplacer la coopération internationale, il serait intéressant d'en examiner la faisabilité en Suisse.

\section{Soutenir les transferts financiers des migrants pour le développement}

Ces dernières années, nombreuses sont les études à avoir mis en évidence l'importance des transferts de fonds des migrants et l'effet positif de certains d'entre eux sur le développement et la lutte contre la pauvretée ${ }^{33}$. A la suite d'une étude mandatée sur les envois financiers des migrants vers la Serbie, le SECO évalue les possibilités de coopération dans ce domaine ${ }^{34}$. La Norvège a lancé un projet pilote pour soutenir les transferts de fonds des migrants au Pakistan: à partir d'un versement de 20000 francs d'un réseau de migrants pakistanais, l'Etat double la mise en ajoutant 20000 francs $^{35}$. Ce système fait penser au système mexicain Tres por uno, qui permet à des associations de migrants aux EtatsUnis de tripler la mise lors d'un transfert, avec un apport équivalent des migrants, de l'Etat local au Mexique et de l'Etat central. Pourquoi ne pas imaginer en effet un apport complémentaire de ce type, mais versé par l'Etat suisse? Ou encore, pourquoi ne pas trouver les moyens de faire baisser les coûts de transaction des transferts financiers? Mais, outre cette baisse des coûts, la coopération au développement doit aussi aider les communautés de migrants qui le souhaitent à mettre sur pied des projets concrets dans leurs régions d'ori-

32 Tamara Keating, «Migration et développement pour l'Afrique: mobiliser les ressources de la diaspora pour le renforcement des capacités dans les pays d'origine», in Schweizerisches Rotes Kreuz (Hrsg.), Migration, ein Beitrag zur Entwicklung ?, Zürich, Seismo, 2007. Voir aussi le site du Programme MIDA Grands Lacs, <http://www.belgium.iom.int/Mida2/presentation.asp>.

33 Voir, dans ce volume, la contribution de Claude Auroi et celle d'Alessandro Monsutti sur les hawâla.

34 Sur les transferts de fonds des migrants serbes établis en Suisse, voir aussi la contribution de Mathias Lerch dans ce volume.

35 Der Bund, 22 juillet 2008. 
gine pour y soutenir l'entrepreneuriat, la création de petites entreprises ou l'accès aux crédits pour des projets locaux encore isolés. Il ne s'agit pas pour l'Etat de s'approprier la gestion de flux financiers privés, mais de soutenir les initiatives des migrants en faveur de leur région d'origine.

\section{Les partenariats migratoires, une panacée?}

La nouvelle Loi fédérale sur les étrangers du 16 décembre 2005 stipule à son article 100 que «le Conseil fédéral encourage les partenariats bilatéraux et multilatéraux avec d'autres Etats dans le domaine des migrations. Il peut conclure des accords visant à renforcer la coopération dans le domaine migratoire et à lutter contre la migration illégale et ses conséquences négatives ». En outre, la mise sur pied de «partenariats migratoires» est censée améliorer le dialogue entre la Suisse et le pays d'origine ou de transit des migrants ${ }^{36}$.

Reste à souhaiter que les mesures envisagées permettent de mieux tenir compte des intérêts des pays d'origine des migrants et ne soient pas à nouveau axées sur les moyens de freiner les migrations. En effet, on parle du renforcement policier et institutionnel dans le pays partenaire pour l'aider à mieux gérer les flux migratoires, ou d'aider les pays du Maghreb à mieux faire face à la migration de transit dans leur pays; la stratégie adoptée par le Conseil fédéral à l'égard des Balkans occidentaux (Kosovo, Bosnie-et-Herzégovine et Serbie) mentionne des mesures visant le renforcement des autorités locales et nationales dans la gestion de la migration, de la lutte contre le trafic des migrants et de l'aide au retour. Mais comment réagiront les représentants de la Suisse lorsque les représentants des pays partenaires réclameront des possibilités de séjour temporaire pour leurs ressortissants à des fins professionnelles? Comment réagiront-ils lorsque les pays d'origine revendiqueront une meilleure lutte en Suisse contre l'exploitation des travailleurs migrants? Il est important qu'un meilleur dialogue s'instaure entre les pays d'origine des migrants et la Suisse.

\section{Conclusion}

La coopération au développement a été trop souvent considérée comme un moyen parmi d'autres de freiner au maximum les flux de migrants arrivant en Suisse et d'encourager les requérants d'asile déboutés à rentrer «volontairement» dans leur pays. Or, vouloir empêcher les flux migratoires s'avère illusoire. Il est temps de concevoir des stratégies de coopération et des politiques migratoires qui prennent mieux en compte la complexité des phénomènes migratoires et leurs aspects positifs.

En Suisse, il faut améliorer le dialogue entre l'administration fédérale, les instances cantonales et la société civile concernée (associations de migrants, ONG de défense des droits humains et ONG de coopération et d'aide humanitaire) et renforcer la participation des associations de migrants au dialogue national et international, non seulement dans la perspective de leur intégration, mais aussi en relation avec leur région ou pays d'origine.

36 Voir la contribution de Therese Liechti et Monica Budowski dans ce volume. 
Dans les pays du Nord, le regard sur le phénomène migratoire est avant tout négatif. La coopération au développement peut favoriser une approche beaucoup plus constructive de la problématique, par des projets concrets d'aide aux migrants, par le plaidoyer et par la prise en considération de la complexité des flux migratoires, tout en luttant contre leurs effets indésirables.

\section{Bibliographie}

Dayton-Johnson, Jeff et Louka T. Katseli, Migration, aide et commerce: plus de cohérence en faveur du développement, Cahier de politique économique, $\mathrm{n}^{\circ}$ 28, Paris, OCDE, 2006.

DDC, Coopération internationale et migrations, Ecrits sur le développement, $\mathrm{n}^{\circ} 4$, Berne, DDC, avril 2001

DDC, Migrations : au-delà des défis, un potentiel à exploiter, dossier, Un seul monde, $\mathrm{n}^{\circ}$ 1, 2008.

DDC, Migrations et coopération au développement, dossier, Un seul monde, $\mathrm{n}^{\circ} 2,1999$.

Longchamp, Claude, Monia Aebersold, Bianca Rousselot und Silvia Ratelband-Pally, Sans Papiers in der Schweiz: Arbeitsmarkt, nicht Asylpolitik ist entscheidend. Schlussbericht im Auftrag des Bundesamtes für Migration, Bern, GFS, Februar 2005.

Moret, Joëlle, Denise Efionayi et Fabienne Stants, Diaspora sri lankaise en Suisse, Berne, ODM, 2007, $156 \mathrm{p}$.

OCDE, La cohérence des politiques au service du développement: migrations et pays en développement, Paris, OCDE, 2007, 164 p.

ODM, Rapport sur la migration 2007, Berne, ODM, avril 2008.

OIM, Enquête sur les partenariats migratoires : présentation du concept dans les contextes international et suisse, Berne, OIM, 2007, 81 p.

Schweizerisches Rotes Kreuz (Hrsg.), Migration, ein Beitrag zur Entwicklung ?, Zürich, Seismo, 2007.

\section{Sites Internet}

Commission mondiale sur les migrations internationales : <http://www.gcim.org >.

DFAE, DDC, présentation du thème de la migration et de quelques projets : <http://www.ddc.admin.ch/ fr/Accueil/Themes/Migrations $>$.

DFAE, DP IV, présentation de l'action dans le domaine des migrations : <http://www.eda.admin.ch/eda/ fr/home/topics/migr.html>.

DFJP, ODM, aide au retour:

<http://www.bfm.admin.ch/bfm/fr/home/themen/rueckkehr/rueckkehrfoerderung.html>. 